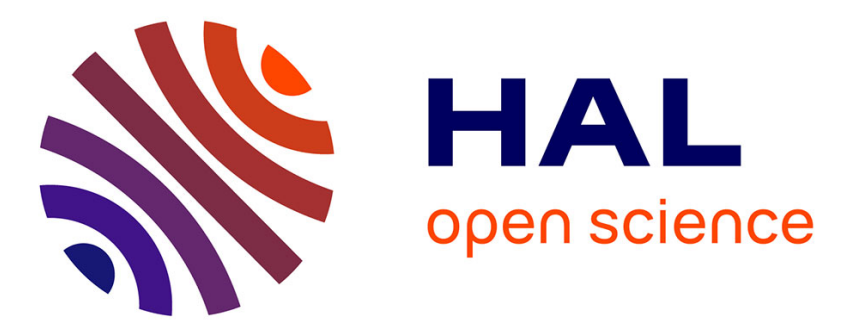

\title{
Interplanetary and interstellar dust observed by the Wind/WAVES electric field instrument
}

\author{
D. Malaspina, M. Horányi, A. Zaslavsky, K. Goetz, L. Wilson, K. Kersten
}

\section{To cite this version:}

D. Malaspina, M. Horányi, A. Zaslavsky, K. Goetz, L. Wilson, et al.. Interplanetary and interstellar dust observed by the Wind/WAVES electric field instrument. Geophysical Research Letters, 2014, 41 (2), pp.266-272. 10.1002/2013GL058786 . hal-02612947

\section{HAL Id: hal-02612947 https://hal.science/hal-02612947}

Submitted on 12 Nov 2021

HAL is a multi-disciplinary open access archive for the deposit and dissemination of scientific research documents, whether they are published or not. The documents may come from teaching and research institutions in France or abroad, or from public or private research centers.
L'archive ouverte pluridisciplinaire HAL, est destinée au dépôt et à la diffusion de documents scientifiques de niveau recherche, publiés ou non, émanant des établissements d'enseignement et de recherche français ou étrangers, des laboratoires publics ou privés.

$$
\text { Copyright }
$$




\section{Geophysical Research Letters}

\author{
RESEARCH LETTER \\ 10.1002/2013GL058786
Key Points:
- First dust detection by Wind is reported
- Dust impact direction can be deter- mined with a new technique
- Wind detects dust in the micron size range

\section{Correspondence to:}

D. M. Malaspina,

David.Malaspina@colorado.edu

\section{Citation:}

Malaspina, D. M., M. Horanyi, A. Zaslavsky, K. Goetz, L. B. Wilson III, and K. Kersten (2014), Interplanetary and interstellar dust observed by the Wind/WAVES electric field instrument, Geophys. Res. Lett., 41, 266-272, doi:10.1002/2013GL058786.

\section{Received 20 NOV 2013}

Accepted 5 JAN 2014

Accepted article online 8 JAN 2014

Published online 28 JAN 2014

Corrected article 21 MAR 2014

The nature of the mechanism that couples the antennas to the impact generated plasma cloud is presently not understood. The previously proposed mechanism of charge recollection by the antennas is not a satisfactory explanation. Therefore, all the parts of the text concerning this mechanism have been removed. The particle size is now inferred by reporting the observed flux on a classical dust flux model : a paragraph explaining this point has been added to the section 4 .

\section{Interplanetary and interstellar dust observed by the Wind/WAVES electric field instrument}

\author{
D. M. Malaspina ${ }^{1}$, M. Horányi ${ }^{1,2}$, A. Zaslavsky ${ }^{3}$, K. Goetz ${ }^{4}$, L. B. Wilson III $^{5}$, and K. Kersten ${ }^{4}$
}

${ }^{1}$ Laboratory for Atmospheric and Space Physics, University of Colorado, Boulder, Boulder, Colorado, USA, ${ }^{2}$ Department of Physics, University of Colorado, Boulder, Boulder, Colorado, USA, ${ }^{3}$ LESIA, Observatoire de Paris, CNRS, Université Pierre et Marie Curie, Université Denis Diderot, Meudon, France, ${ }^{4}$ School of Physics and Astronomy, University of Minnesota, Minneapolis, Minnesota, USA, ${ }^{5}$ NASA Goddard Space Flight Center, Greenbelt, Maryland, USA.

\section{Introduction}

Hypervelocity $(v>1 \mathrm{~km} / \mathrm{s}$ ) dust impacts on spacecraft produce transient clouds of impact-generated plasma which can be measured by spaceborne electric field instruments as brief, high-amplitude voltage spikes. Typical spikes have durations on the order of milliseconds and amplitudes from a few to hundreds of $\mathrm{mV} / \mathrm{m}$. The Voyager spacecraft measured such spikes in the ring plane of Saturn [Gurnett et al., 1983], as did Cassini [Kurth et al., 2006]. The Vega and Deep Space 1 spacecraft observed similar spikes during cometary encounters [Laakso et al., 1989; Tsurutani et al., 2004]. Most recently, the twin STEREO spacecraft orbiting the Sun at $1 \mathrm{AU}$ have observed such spikes associated with the impacts of both micrometer and nanometer radius dust [Meyer-Vernet et al., 2009; Zaslavsky et al., 2012].

In this work, dust impacts on the Wind spacecraft are reported for the first time, measured by the Wind/WAVES electric field instrument. Dust impact direction is directly determined for the first time by a spacecraft measuring dust with electric field data. The impact direction determination method is described in section 3.

Two primary populations of dust are known to exist at $1 \mathrm{AU}$, interplanetary dust (IPD) and interstellar dust (ISD). IPD with typical radii of $\simeq 1 \mu \mathrm{m}$ migrate toward the Sun due to Poynting-Robertson drag. These particles are expected to follow approximately circular Keplerian orbits, providing a constant impact rate with relatively modest impact speeds on the order of $\simeq 1 \mathrm{~km} / \mathrm{s}$. These grains break up in collisions, and erode due to sublimation and sputtering near the Sun. Their debris with characteristic radii $\simeq 0.1 \mu \mathrm{m}$ are the so-called $\beta$ meteorites, for which radiation pressure overcomes solar gravity. These particles are expected to impact Wind throughout a given year with typical relative speeds of $>10 \mathrm{~km} / \mathrm{s}$ [Grün et al., 1985]. Smaller debris with radii $\ll 0.1 \mu \mathrm{m}$, so-called nanograins, get entrained in the solar wind and can reach impact speeds $>100 \mathrm{~km} / \mathrm{s}$ [Meyer-Vernet et al., 2009]. Their fluxes are highly variable since their motion is strongly affected by the instantaneous configuration of the interplanetary magnetic fields inside $1 \mathrm{AU}$ [Juhász and Horányi, 2013].

ISD was first detected by the Ulysses dust detector, which measured impacts from a direction opposite to that expected for interplanetary dust grains with impact velocities exceeding the local solar system escape velocity [Grün et al., 1993]. Subsequent analysis showed that Ulysses' orbital plane was nearly orthogonal to a flow of ISD particles, moving through the solar system approximately parallel to the flow of neutral interstellar hydrogen and helium gas, traveling at $\sim 26 \mathrm{~km} / \mathrm{s}$. ISD motion within the heliosphere is determined by gravity, electromagnetic forces, and radiation pressure. ISD particles that penetrate to $1 \mathrm{AU}$ near the 



Figure 1. (left) Schematic of Wind antenna geometry. (right) The differential potential measured by the $E_{x}\left(E_{y}\right)$ dipole during a 17 ms TDS waveform capture on 25 December 2007.

ecliptic plane are thought to have primarily micron radii [Mann et al., 2000; Landgraf et al., 2003]. ISD flux for $0.15 \mu \mathrm{m}$ to $0.45 \mu \mathrm{m}$ radius particles was observed to be $2.5 \times 10^{-5} \mathrm{~m}^{-2} \mathrm{~s}^{-1}$ by Cassini near $1 \mathrm{AU}$ in the ecliptic plane [Altobelli et al., 2003]. An ISD flux of approximately micron radius particles was observed to be $2.6 \times 10^{-6} \mathrm{~m}^{-2} \mathrm{~s}^{-1}$ by Helios between 0.3 and 1 AU [Altobelli et al., 2006].

In the following analysis, dust flux and impact directionality are used to demonstrate that Wind observes both IPD and ISD with approximately micron radii. Nanometer dust is not detected by Wind when STEREO and Wind are in close proximity and STEREO observes nanodust (October 2006-February 2007), even though both spacecraft have similar electric field waveform capture capability. Dust impact directionality and flux are consistent with STEREO's results on ISD [Zaslavsky et al., 2012]. Regarding IPD, Wind's observations are consistent with an observed interplanetary micron dust population dominated by particles on bound orbits, rather than hyperbolic $\beta$ meteoroids as suggested by STEREO data analysis.

\section{Dust on Wind}

Wind is a spin-stabilized spacecraft, $1.8 \mathrm{~m}$ in height and $2.4 \mathrm{~m}$ in diameter. The cylindrical body of Wind is covered in solar panels, while the top and bottom surfaces are covered with multilayer insulation. The spacecraft spin plane differs from the ecliptic plane by $<1^{\circ}$. Wind has six electric field sensors: four spin plane fine wire antennas $\left(E_{x}^{+}, E_{x}^{-}, E_{y}^{+}, E_{y}^{-}\right)$and two axial cylindrical antennas $\left(E_{z}^{+}, E_{z}^{-}\right)$. These sensors are operated as three orthogonal dipoles $\left(E_{x}, E_{y}, E_{z}\right)$, where the voltage difference between opposing antenna pairs is measured: $v_{x}=\left(V_{x}^{+}-V_{x}^{-}\right), v_{y}=\left(V_{y}^{+}-V_{y}^{-}\right)$, and $V_{z}=\left(V_{z}^{+}-V_{z}^{-}\right)$. Each $V_{i}^{+/-}$represents the voltage (relative to spacecraft ground) measured by antenna $E_{i}^{+/-}$. Soon after launch the physical tip-to-tip dipole lengths were $101.8 \mathrm{~m}, 16.8 \mathrm{~m}$, and $10.56 \mathrm{~m}$ for the $E_{x}, E_{y}$, and $E_{z}$ dipoles, respectively. The left side of Figure 1 shows a schematic of the Wind antennas in the ecliptic plane (not to scale). Soon after launch, the dipole electrical lengths were $L_{X}=41 \mathrm{~m}, L_{Y}=3.8 \mathrm{~m}$, and $L_{Z}=2.2 \mathrm{~m}$. The $E_{X}^{+}$antenna was cut twice during the Wind mission, possibly due to dust impacts. The electrical length of the $E_{x}$ dipole was reduced to $27 \mathrm{~m}$ after the first cut on 3 August 2000, and to $25 \mathrm{~m}$ after the second cut on 24 September 2002, suggesting that the first cut shortened $E_{x}^{+}$substantially, while the second cut removed only a small section. Spacecraft orbital data was used to determine that both breaks occurred on $E_{x}^{+}$.

This study examines data from the Wind/WAVES Time Domain Sampler (TDS) [Bougeret et al., 1995] fast stream, which samples electric field data at $120 \mathrm{kHz}$, producing waveform snapshots $17 \mathrm{~ms}$ in duration, $\approx 100$ of which are telemetered to Earth per day. Events are chosen by an onboard algorithm that preferentially selects high- amplitude electric field signals from the $E_{x}$ data stream. The TDS returns data from only 


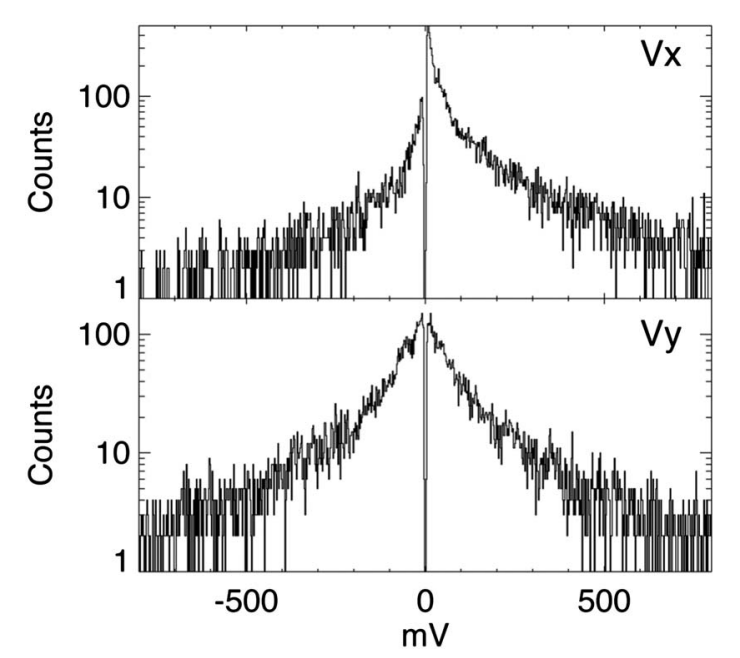

Figure 2. Histograms of the value furthest from 0 during each dust waveform for differential antenna potentials (top) $V_{x}$ and (bottom) $V_{y}$.

two of the three dipoles. The operating mode used almost exclusively since launch is to return data from $E_{x}$ and $E_{y}$ while using $E_{x}$ to trigger TDS captures. Since 2004, Wind has been located at the first Lagrange point (L1) in the solar wind and approximately 180 Earth radii $\left(R_{E}\right)$ upstream of the Earth.

The rightmost panels of Figure 1 show a typical dust impact waveform recorded by the TDS. These signals characteristically show a fast rise (positive or negative), followed by a slower decay. Similar signals appear in electric field data on other spacecraft [Gurnett et al., 1983; Kurth et al., 2006; Zaslavsky et al., 2012].

Wind/TDS data recorded between 1 January 2006 and 31 December 2009 are examined. Dust impact waveforms are identified by the following procedure: (1) TDS captures with $\operatorname{Max}\left(\left|V_{y}\right|\right)<4 \mathrm{mV}$ are excluded. This removes most electrostatic solitary waves. (2) Events are excluded if $\operatorname{Max}\left(\left|V_{y}\right|\right) / \operatorname{RMS}\left(V_{y}\right)<4$. This removes events without sharp spikes. (3) Remaining TDS captures are visually inspected to ensure that only waveforms similar to Figure 1 are retained. The resulting data set contains 14,824 dust impact waveforms, representing $6.2 \%$ of the 240,003 Wind/TDS fast stream captures between 2006 and 2009. Dust impacts often produce signals with high amplitudes compared to most solar wind plasma waves. Therefore, it is likely that most dust impacts capable of producing large voltage spikes are captured by the TDS.

$E_{y}$ dipole data is used to identify dust impacts because the physically asymmetric $E_{x}$ dipole has an asymmetric response to dust impacts and so cannot be used for direction determination. $E_{x}$ dust signals are biased $\left(\left|V_{x}^{+}\right|>\left|V_{x}^{-}\right|\right)$, consistent with a stronger signal on the shorter (cut) antenna. Figure 2 shows histograms for $V_{x}$ (top) and $V_{y}$ (bottom) of the signal value furthest from 0 during each dust waveform identified. The asymmetry on $E_{x}$ is not present on $E_{y}$. The distribution of $V_{y} / V_{x}$ for all impacts has a mean of 3.0 and a median of 1.5. It is likely that shorter antennas ( $E_{x}^{+}$compared to $E_{x}^{-}$or $E_{y}^{+/-}$compared to $E_{x}^{+/-}$) often see larger voltages perhaps because they have a lower free space capacitance than longer antennas.

\section{Observations and Analysis}

The distribution of daily occurrence rates for Wind/TDS dust is well fit by a Gaussian centered on 10.1 hits/day with a standard deviation of 5.2 hits/day during 2006-2009. The cross-sectional area of the Wind spacecraft body perpendicular to the spin axis is approximately $4.3 \mathrm{~m}^{2}$, implying a flux of $(2.7 \pm 1.4) \times$ $10^{-5} \mathrm{~m}^{-2} \mathrm{~s}^{-1}$. The Wind-derived flux is consistent with the micron-sized dust flux derived from STEREO/ WAVES TDS measurements: $1-16 \times 10^{-5} \mathrm{~m}^{-2} \mathrm{~s}^{-1}$ from 2007 to 2010 . This range reflects uncertainty in STEREO's effective collecting area [Zaslavsky et al., 2012]. Wind-observed dust fluxes are also consistent with Ulysses dust measurements [Grün et al., 1985], assuming a mass threshold $\simeq 10^{-14} \mathrm{~kg}$, or silica particles with radii $\simeq 1 \mu \mathrm{m}$.

Nanodust observed by STEREO showed average fluxes roughly between $2 \times 10^{-3}$ and $10 \mathrm{~m}^{-2} \mathrm{~s}^{-1}$ in the period 2007-2010 [Zaslavsky et al., 2012]. Dust fluxes observed by Wind are orders of magnitude smaller, demonstrating that nanodust is not observed by Wind, even though Wind was within $300 R_{E}$ of both STEREO 


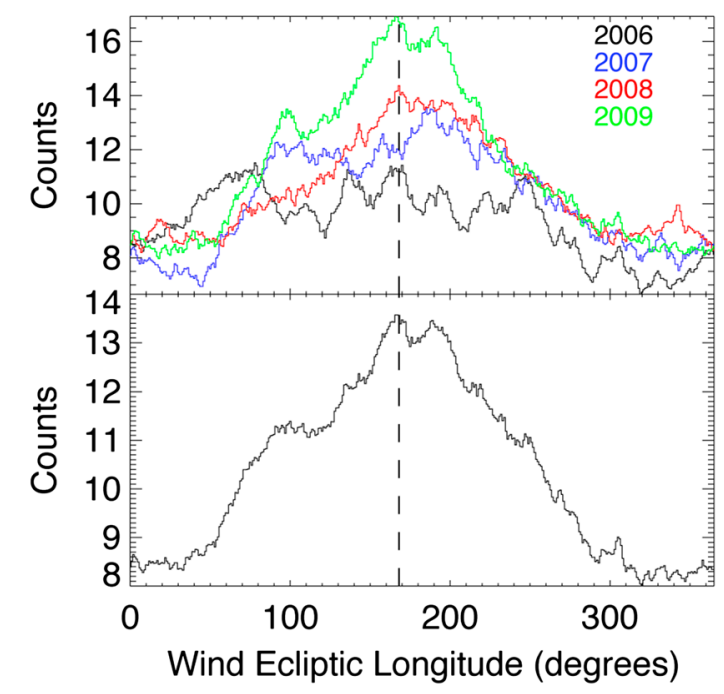

Figure 3. Dust impacts per day binned by ecliptic longitude and smoothed. (top) Each studied year and (bottom) the average over these 4 years. The vertical dashed line indicates $168^{\circ}$ ecliptic longitude.

spacecraft from October 2006 to February 2007, a time when STEREO observed high nanodust fluxes. This difference may be due to differences in spacecraft surface materials or the coupling mechanism between the antennas and the charge released during the impact. This mechanism is not yet properly understood in the case of WIND, but it seems that unlike STEREO, large grains are necessary to produce detectable signals.

Figure 3 shows the number of Wind-observed dust impacts per day, binned by ecliptic longitude and smoothed using a 40 day boxcar window. The top panel shows each year separately and the bottom panel shows the average of all four years. The vertical dashed line indicates $168^{\circ}$ ecliptic longitude. ISD flows from approximately the same direction as the interstellar neutral gas: ecliptic longitude $\lambda=258 \pm 20^{\circ}$ and latitude $\theta=8 \pm 10^{\circ}$ with a velocity of $\approx 26 \mathrm{~km} / \mathrm{s}$ [Grün et al., 1993]. The observed ISD flux is expected to be greatest when Wind's orbital velocity about the Sun $(\sim 30 \mathrm{~km} / \mathrm{s})$ is antiparallel to the ISD velocity, creating the highest relative velocity between Wind and the ISD flow. This occurs at $258^{\circ}-90^{\circ}=168^{\circ}$, on about 9 March. During this time, ISD impacts should more frequently result in the large voltage spikes that are preferentially selected by the TDS. Conversely, when the Wind orbital velocity is parallel to the ISD velocity, the relative velocity is lowest and the flux of measurable ISD should decrease. This occurs near ecliptic longitude of $348^{\circ}$ on about 12 September.

The smoothed dust count data in Figure 3 peaks approximately at $168^{\circ}$ for 2009 , and near $190^{\circ}$ for 2007 and 2008. Variation in dust detection as a function of ecliptic longitude is consistent with ISD detection. The Wind/WAVES results validate the STEREO/WAVES electric field measurements of micron dust that also showed an ISD-related peak near ecliptic longitude of $168^{\circ}$ [Zaslavsky et al., 2012]. Data from 2006 (not measured by STEREO) is significantly different. The dust is close to isotropic in longitude and the overall count rate is lower, indicating a significant deviation from expected ISD behavior.

The TDS data collection capability and rotating spacecraft allow Wind, uniquely among spacecraft flown to date, to directly determine the impact direction of dust impacts using electric field data. To determine impact direction, it is assumed that the antenna closest to a given impact location observes a larger positive voltage spike than the opposing antenna. This choice will be justified a posteriori. With this convention, the negative $V_{y}$ spike in Figure 1 implies that the impact was closer to $E_{y}^{-}$than $E_{y}^{+}$. In this way, one can identify which half of the Wind spacecraft experienced a given dust impact. Wind spins at 20 rotations per minute, exposing the center of one half of the spacecraft body to a given look direction every $3 \mathrm{~s}$. Because the response of Wind's antennas to dust impacts as a function of the distance between the impact location and antenna base is unknown, all angles $\pm 90^{\circ}$ from the antenna closest to the impact site are assigned an equal probability of impact. A pseudocount distribution as a function of impact angle is constructed by adding 1 pseudocount per degree per dust impact over the 180 degrees corresponding to the impacted half. Wind's Sun sensors provide knowledge of the spacecraft rotation angle with respect to the Sun for each dust hit. 



Figure 4. Monthly histograms of averaged pseudocounts versus degrees away from the expected interstellar dust impact direction (see text for detail). The central figure shows Wind's location about the Sun each month. Green, red, and blue lines show Earth's motion, the sunward direction, and the expected interstellar dust flow direction, respectively. Colored lines on the pseudocount distributions retain their meanings.

Figure 4 shows monthly pseudocount distributions. The data for each month is averaged over 2006-2009. Horizontal axes indicate degrees clockwise from the expected ISD flow direction. Vertical axes are pseudocounts, as described in the preceding paragraph. Green lines show the ram direction of Wind about the Sun each month. Blue lines indicate the expected ISD flow direction. The number in the lower right of each plot indicates the difference between the maximum and minimum of that month's pseudocount distribution. The central figure shows the position of Wind (green star) about the Sun (yellow star) on the fifteenth of each month. Green and blue lines retain their meanings. Red lines point sunward. The relative velocity between Wind and an approximately isotropic interplanetary dust population is expected to peak the ram direction, leading to a maximum in the observed flux, because Wind dust observation depends strongly on impact velocity.

For each month, the averaged pseudocount distribution peak is near the spacecraft ram direction and highly variable ( $180 \pm 36$ counts). Pseudocount distribution minima occurs in the antiram direction, and are less variable $(129 \pm 21$ counts). When the distribution peak is not in the ram direction, it is skewed toward the expected direction of ISD flow. When Earth's orbital velocity and interstellar flow velocity oppose in March, the distribution peak increases, as does the difference between the peak and the minimum. This is also observed to a lesser extent in February and April. Near September, when Earth's orbital velocity and the ISD velocity are nearly parallel, dust is distributed more uniformly with impact angle. Both the distribution peak and the difference between peak and minimum become small. This is also true for August and October. These properties indicate that the dust observed by Wind is primarily IPD, with an ISD component modulated by Wind's velocity relative to the ISD flow. IPD flux is omnidirectional, but modulated by the ram direction of Wind with respect to the Sun, suggesting that particles on elliptical trajectories crossing Earth's orbit, such as the dust trails of comets, are the dominant contribution to the detected IPD. The ram modulation is inconsistent with $\beta$ meteoroids or other particles on hyperbolic orbits, which would be expected to impact Wind primarily between the ram and sunward directions. Additionally, the single-month pseudocount plots averaged to produce Figure 4 have strong enhancements in IPD flux in specific look directions on one to several day timescales (not plotted), which may conceivably be remnants from collisions of larger bodies traveling on similar orbits. 


\section{Discussion and Conclusions}

Hypervelocity dust impacts and their impact direction on the Wind spacecraft were reported here for the first time. Dust flux and directionality indicated micron-sized dust of both interplanetary and interstellar origin. While these observations are consistent with prior reports of micron-sized IPD and ISD by the Ulysses dust detector and STEREO/WAVES [Zaslavsky et al., 2012], Wind-observed dust flux was found to be inconsistent with reported nanodust observations by STEREO [Meyer-Vernet et al., 2009; Zaslavsky et al., 2012] when the spacecraft were in close proximity. Both Wind and STEREO possess similar electric field waveform capture electronics, but significantly different antenna geometry and spacecraft surface materials. The lack of nanodust detection by Wind therefore suggests that one of these two differences accounts for STEREO's so far unique ability to detect nanodust at $1 \mathrm{AU}$ using electric field antennas.

The actual mechanism through which dust impacts generate the signals detected by Wind/WAVES is presently not well understood. The fact that the instrument operates in dipole mode and results of the directionality analysis provide two clear constraints on this mechanism: it must generate a much stronger pulse on the antenna close to the impact than on the opposite one (otherwise, no signal would be detected by the dipole), and the voltage pulse must be positive. Future investigations to elucidate the nature of the coupling mechanism will need to take these points into account.

Even in the absence of the knowledge of a mechanism that would allow us to perform a direct mass calibration, it is possible to derive information on the size of the detected grains on the basis of their observed flux. Reporting on the flux model presented in Grün et al. [1985], the value $1.3-4.1 \times 10^{-5} \mathrm{~m}^{-2} \mathrm{~s}^{-1}$ observed by Wind, one can derive a mass range $5 \times 10^{-16} \mathrm{~kg}-1.2 \times 10^{-14} \mathrm{~kg}$ for the smallest detected grain (value at which the cumulative flux is calculated). It corresponds to a size roughly between 0.3 and $1 \mu \mathrm{m}$, assuming a mass density $\rho=2.5 \mathrm{~g} / \mathrm{cm} 3$.

Wind ISD observations indicate significant changes in flux and flow direction at 1 AU from year to year. Based on Figure 3, ISD comprised $\approx 18 \%, 30 \%, 36 \%$, and $47 \%$ of the total dust flux observed in March of 2006, 2007,2008 , and 2009 , respectively. The estimated peak interstellar flow direction changed by $\approx 25^{\circ}$ between March 2008 and March 2009 (Figure 3). Observations by Ulysses at larger solar radial distances (1-5 AU) between 1992 and 2007 also show significant variability in ISD flux and flow direction. At times, the ISD flow deviated from the interstellar gas flow by up to $50^{\circ}$ [Krüger et al., 2007; Krüger and Grün, 2009; Strub et al., 2011]. The variable flux and direction of ISD particles may be due to variability in solar radiation pressure, as well as the variability of the large-scale heliospheric magnetic fields through solar cycles [Landgraf et al., 2003; Krüger et al., 2007; Sterken et al., 2013]. Because the Wind dust data set overlaps with the years of Ulysses operation, it can provide a second spatial data point (at $1 \mathrm{AU}$ ) against which ISD propagation models can be calibrated.

Probability distributions of dust impact locations on the Wind spacecraft body in the ecliptic plane indicated that IPD is primarily observed in the spacecraft ram direction with respect to its orbit about the Sun, suggesting that the IPD detected by Wind is likely dominated by dust on bound trajectories crossing Earth's orbit, rather than $\beta$ meteoroids as suggested by Zaslavsky et al. [2012]. Dust in the antiram direction (most consistent with IPD) showed significantly less variation than dust in the ram direction (consistent with IPD plus ISD modulated by Wind's orbit). Flux enhancements in specific look directions over one to several days are present in the data, potentially indicative of debris trails from larger bodies traveling on similar orbits.

Correspondence between Wind data and both ram and ISD dust flow directions was achieved by assuming that the antenna closest to the impact location observes a higher positive potential than the opposing antenna. This argues against recollection of impact plasma electrons by the spacecraft body as the primary mechanism for creating the measured voltage. Body recollection would produce a common signal on opposing antennas that would be largely removed because Wind operates in dipole mode. The mechanism coupling the measured voltages to the impact plasma must instead produce an asymmetric response on opposing electric field antennas. While determining the exact coupling mechanism is beyond the scope of this work, differences in antenna geometry and dust signals between Wind and STEREO may provide new insight. A reasonable calibration between measured voltages and impact charge can likely be achieved on Wind, as it has been on STEREO [Zaslavsky et al., 2012].

Wind has orbited the Sun at 1 AU for nearly 20 years, accumulating a large and, until this work, unexplored set of dust observations. The temporal span of this data set, combined with the unique ability of Wind 


\section{Acknowledgments}

The authors thank the Wind/WAVES team for their continued support with data retrieval and calibration, especially P.J. Kellogg. This work was supported by NASA award NNX10AP73G.

The Editor thanks Sheng-Yi Ye and an anonymous reviewer for their assistance in evaluating this paper. to determine dust impact direction using electric field antennas, will enable new characterization of the dynamic micron dust environment at $1 \mathrm{AU}$ on timescales from a few days to nearly two solar cycles.

\section{References}

Altobelli, N., S. Kempf, M. Landgraf, R. Srama, V. Dikarev, H. Krüger, G. Moragas-Klostermeyer, and E. Grün (2003), Cassini between Venus and Earth: Detection of interstellar dust, J. Geophys. Res., 108(A10), 8032, doi:10.1029/2003JA009874.

Altobelli, N., E. Grün, and M. Landgraf (2006), A new look into the Helios dust experiment data: Presence of interstellar dust inside the Earth's orbit, Astron. Astrophys., 448, 243-252, doi:10.1051/0004-6361:20053909.

Bougeret, J.-L., et al. (1995), WAVES: The radio and plasma wave investigation on the Wind spacecraft, Space Sci. Rev., 71, 231-263, doi:10.1007/BF00751331.

Grün, E., H. A. Zook, H. Fechtig, and R. H. Giese (1985), Collisional balance of the meteoritic complex, Icarus, 62, 244-272, doi:10.1016/0019-1035(85)90121-6.

Grün, E., et al. (1993), Discovery of Jovian dust streams and interstellar grains by the ULYSSES spacecraft, Nature, 362, 428-430, doi:10.1038/362428a0.

Gurnett, D. A., E. Grun, D. Gallagher, W. S. Kurth, and F. L. Scarf (1983), Micron-sized particles detected near Saturn by the Voyager plasma wave instrument, Icarus, 53, 236-254, doi:10.1016/0019-1035(83)90145-8.

Juhász, A., and M. Horányi (2013), Dynamics and distribution of nanodust particles in the inner solar system, Geophys. Res. Lett., 40, 2500-2504, doi:10.1002/grl.50535.

Krüger, H., and E. Grün (2009), Interstellar dust inside and outside the heliosphere, Space Sci. Rev., 143, 347-356, doi:10.1007/s11214-008-9431-3.

Krüger, H., M. Landgraf, N. Altobelli, and E. Grün (2007), Interstellar dust in the solar system, Space Sci. Rev., 130, 401-408, doi:10.1007/s11214-007-9181-7.

Kurth, W. S., T. F. Averkamp, D. A. Gurnett, and Z. Wang (2006), Cassini RPWS observations of dust in Saturn's E Ring, Planet. Space Sci., 54, 988-998, doi:10.1016/j.pss.2006.05.011.

Laakso, H., R. Grard, A. Pedersen, and G. Schwehm (1989), Impacts of large dust particles on the VEGA spacecraft, Adv. Space Res., 9 , 269-272, doi:10.1016/0273-1177(89)90273-1.

Landgraf, M., H. Krüger, N. Altobelli, and E. Grün (2003), Penetration of the heliosphere by the interstellar dust stream during solar maximum, J. Geophys. Res., 108(A10), 8030, doi:10.1029/2003JA009872.

Mann, I., A. Krivov, and H. Kimura (2000), Dust cloud near the Sun, Icarus, 146, 568-582, doi:10.1006/icar.2000.6419.

McBride, N., and J. A. M. McDonnell (1999), Meteoroid impacts on spacecraft: Sporadics, streams, and the 1999 Leonids, Planet. Space Sci., 47, 1005-1013, doi:10.1016/S0032-0633(99)00023-9.

Meyer-Vernet, N., et al. (2009), Dust detection by the Wave instrument on STEREO: Nanoparticles picked up by the solar wind?, Sol. Phys., 256, 463-474, doi:10.1007/s11207-009-9349-2.

Sterken, V. J., N. Altobelli, S. Kempf, H. Krüger, R. Srama, P. Strub, and E. Grün (2013), The filtering of interstellar dust in the solar system, Astron. Astrophys., 552, 1-22, doi:10.1051/0004-6361/201219609.

Strub, P., V. J. Sterken, H. Krüger, E. Grün, and M. Horanyi (2011), Interstellar dust flow through the solar system, in American Institute of Physics Conference Series, American Institute of Physics Conference Series, vol. 1397, edited by V. Y. Nosenko et al., pp. 385-386, AIP Publishing, Melville, NY, doi:10.1063/1.3659855.

Tsurutani, B. T., D. R. Clay, L. D. Zhang, B. Dasgupta, D. Brinza, M. Henry, J. K. Arballo, S. Moses, and A. Mendis (2004), Plasma clouds associated with Comet P/Borrelly dust impacts, Icarus, 167, 89-99, doi:10.1016/j.icarus.2003.08.021.

Zaslavsky, A., et al. (2012), Interplanetary dust detection by radio antennas: Mass calibration and fluxes measured by STEREO/WAVES, J. Geophys. Res., 117, A05102, doi:10.1029/2011JA017480. 